\title{
Asymptotic safety in the sine-Gordon model
}

\author{
J. Kovács, S. Nagy, ${ }^{*}$ and K. Sailer \\ Department of Theoretical Physics, University of Debrecen, P.O. Box 5, H-4010 Debrecen, Hungary
}

(Received 12 August 2014; published 23 February 2015)

\begin{abstract}
In the framework of the functional renormalization group method, it is shown that the phase structure of the two-dimensional sine-Gordon model possesses a nontrivial UV fixed point which makes the model asymptotically safe. The fixed point exhibits strong singularity similar to the scaling found in the vicinity of the infrared fixed point. The singularity signals the upper energy-scale limit to the validity of the model. We argue that the sine-Gordon model with a momentum-dependent wave-function renormalization is in a dual connection with the massive sine-Gordon model.
\end{abstract}

DOI: 10.1103/PhysRevD.91.045029

PACS numbers: 11.10.Gh, 05.10.Cc, 11.10.Hi

\section{INTRODUCTION}

The two-dimensional (2D) sine-Gordon (SG) model in Euclidean spacetime is one of the most important scalar models [1]. It has significant relevance starting from low energy condensed matter systems [2], where it serves as an effective theory up to supersymmetry $[3,4]$ or string theory [5]. The model is integrable [6-9]; therefore, it is possible to get analytic information from its physical properties.

The functional renormalization group (RG) treatment [10-14] for the SG model is capable of describing the phase structure and identifying the Kosterlitz-Thouless (KT) fixed point $[15,16]$ of the model even in the local potential approximation [17-19]. The perturbative RG treatment could account for the KT-type essential scaling of the correlation length [20]. The functional RG treatment also can describe the essential scaling in equivalent models $[21,22]$. It is possible to investigate the SG model directly in the framework of the functional RG method by taking into account the evolution of the wave-function renormalization [23-25], where the scaling of the coupling can be traced from the perturbative regime to the infrared (IR) one. It was shown that there exists an IR fixed point $[23,24]$, which appears in the low energy-scaling regime of the broken symmetric phase. There are further models where the IR fixed point was identified successfully [24,26-31].

During the evolution in the IR regime of the broken phase in the SG model, a mass gap is generated. The spectrum of the soliton excitations is exactly known [32,33], so as the $S$ matrix in the model. The latter can be obtained even in the case when one takes into account boundary terms in the potential $[34,35]$. Moreover, the soliton reflection factors on the ground-state boundary can also be determined analytically [36-38]. Uncovering the mass gap in the SG model with the RG technique can raise difficulties, but the problem can be handled by considering the evolution of the field-dependent wave-function renormalization [39]. If we include the

* Corresponding author. nagys@dtp.atomki.hu boundary terms in the potential, then we should be faced with further enormous technical problems due to the increasing number of couplings and the modified symmetry properties; therefore, the solution of the boundary SG model seems not to be available by the RG method. Furthermore, the boundary terms restrict the path integral for the noncompact field variables, which needs the background field technique [40], but this treatment is out of the scope of this work. Wenote that there are attempts to discuss the monotonous decrease of the central charge for the model in the framework of the RG method [9,41,42].

Besides the existing fixed points, it is questionable how the model behaves in the UV region of the symmetric phase. The UV scaling of the coupling is irrelevant there, making it perturbatively nonrenormalizable. We argue that there exists an UV non-Gaussian fixed point (NGFP) in the SG model. Our goal in this article is to clarify the UV scaling behavior of the model. The UV NGFP makes the coupling finite, safe from divergences. This is the main idea of the asymptotic safety [31,43-45]. Nowadays, the asymptotically safe models are the focus in the RG treatment, since they usually cannot be handled by perturbative tools. Recently, the quantum Einstein gravity (QEG) has been one of the most popular models investigated in the framework of the RG method [46-48] and seems to show an UV NGFP, making the model asymptotically safe, even in its extensions [49-53].

There are several extensions of the SG model; the simplest one contains a simple mass term [54-57]. The mass is a relevant coupling in the theory and breaks the periodic symmetry. In the UV limit, the mass dies out; therefore, the model shows an essential scaling. However, in the IR, where the mass becomes more and more important, the essential scaling is replaced by a scaling belonging to a second order phase transition [30].

In this article, we make a further generalization of the SG model by taking into account a momentum-dependent wave-function renormalization. Its Taylor expansion in the momentum provides further couplings. There are couplings which are irrelevant and can cause troubles in the UV. We study how these couplings affect the 
asymptotic safety and how they are related to the massive SG (MSG) model.

The paper is organized as follows. In Sec. II, the investigated models and the RG method are introduced. In Sec. III, we discuss how the asymptotic safety appears in the SG model. The extensions of the SG model are treated in Secs. IV and V. The idea of duality among SG models is sketched in Sec. VI. Finally, in Sec. VII the conclusions are drawn up.

\section{THE SINE-GORDON MODEL}

The functional renormalization group equation for the effective action of a Euclidean field theory is [10]

$$
\dot{\Gamma}_{k}=\frac{1}{2} \operatorname{Tr} \frac{\dot{R}_{k}}{R_{k}+\Gamma_{k}^{\prime \prime}},
$$

where the notations ${ }^{\prime}=\partial / \partial \varphi, \cdot=k \partial_{k}$ are used, and the trace $\mathrm{Tr}$ signals the integration over all momenta. The IR regulator function $R_{k}$ stands for removing the UV and IR divergences if necessary. We have solved Eq. (1) by using the following form of the effective action

$$
\Gamma_{k}=\int_{x}\left[\frac{Z_{k}\left(\partial^{2}\right)}{2}\left(\partial_{\mu} \phi\right)^{2}+V_{k}(\phi)\right]
$$

with the local potential

$$
V_{k}(\phi)=u \cos (\phi),
$$

and the field-independent wave-function renormalization $Z_{k}\left(\partial^{2}\right)$ containing higher derivative terms. The latter becomes momentum dependent in Fourier space and is parametrized as

$$
Z_{k}=z+z_{1} p^{2}
$$

i.e., keeping only the first nontrivial term. The evolution equations for the couplings $u, z$, and $z_{1}$ are

$$
\begin{aligned}
\dot{u}= & \mathcal{P}_{1} \int_{p} k^{2} \mathcal{D} \\
\dot{z}= & 2 \mathcal{P}_{0} \int_{p} k^{2} V^{\prime \prime \prime 2} \mathcal{D}^{4}\left[-\partial_{p^{2}} Z-2 z_{1} p^{2}+2 p^{2}\left(\partial_{p^{2}} Z\right)^{2} \mathcal{D}\right] \\
\dot{z}_{1}= & \mathcal{P}_{0} \int_{p} k^{2} V^{\prime \prime \prime 2} \mathcal{D}^{4}\left[-2 z_{1}\right. \\
& +\left[24 p^{2} z_{1} \partial_{p^{2}} Z+2\left(\partial_{p^{2}} Z\right)^{2}+12 z_{1}^{2} p^{4}\right] \mathcal{D} \\
& -\left[12 p^{2}\left(\partial_{p^{2}} Z\right)^{3}+36 p^{4} z_{1}\left(\partial_{p^{2}} Z\right)^{2}\right] \mathcal{D}^{2} \\
& \left.+12 p^{4}\left(\partial_{p^{2}} Z\right)^{4} \mathcal{D}^{3}\right]
\end{aligned}
$$

with the IR regulator $R_{k}=k^{2}$, the dressed propagator $\mathcal{D}=$ $1 /\left(Z_{k} p^{2}+k^{2}+V_{k}{ }^{\prime \prime}\right)$, and $\mathcal{P}_{n} \ldots=(2 \pi)^{-1} \int_{0}^{2 \pi} d \phi \cos (n \phi) \ldots$ being the projection onto the field-independent subspace.

\section{ASYMPTOTIC SAFETY}

First, we restrict ourselves to consider the RG evolution of the dimensionless couplings $\tilde{u}=u / k^{2}$ and $z$ and keep $\tilde{z}_{1}=0$. The evolution equations are [23]

$$
\begin{aligned}
& \dot{\tilde{u}}=-2 \tilde{u}+\frac{1}{2 \pi \tilde{u} z}\left[1-\sqrt{1-\tilde{u}^{2}}\right], \\
& \dot{z}=-\frac{1}{24 \pi} \frac{\tilde{u}^{2}}{\left(1-\tilde{u}^{2}\right)^{3 / 2}} .
\end{aligned}
$$

The phase space spanned by the couplings $\tilde{u}$ and $z$ are shown in Fig. 1. If we try to look for the fixed points of the flow equations in Eq. (6), then we cannot find any. However, if we Taylor expand the RG equations in the fundamental mode $\tilde{u}$, then at $\tilde{u}^{*}=0$ with arbitrary $z^{*}$, we have a line of fixed points. In a certain sense, the line can be considered as the Gaussian fixed point(s) of the model. Its scaling behavior shows that for $z^{*}>1 / 8 \pi$, the section of the corresponding line is constituted by UV attractive fixed points, while for $z^{*}<1 / 8 \pi$, we have UV repulsive or IR attractive ones. In other words, in the vicinity of the lines of fixed points, the evolution of the fundamental mode $\tilde{u}$ can be either relevant (for $1 / z<8 \pi$ ) or irrelevant (for $1 / z>8 \pi)$. The scaling regimes are separated by the KT point at $\tilde{u}_{c}^{*}=0$ and $z_{c}^{*}=1 / 8 \pi$.

The IR limit of the relevant scaling in the broken phase signals divergences and nonphysical regimes. Similarly, the UV limit of the irrelevant scaling can cause some trouble. Both problems can be solved by finding nontrivial fixed points in these limits.

The IR fixed point was uncovered so far in [23]. It has been shown that the rescaling of the original variables

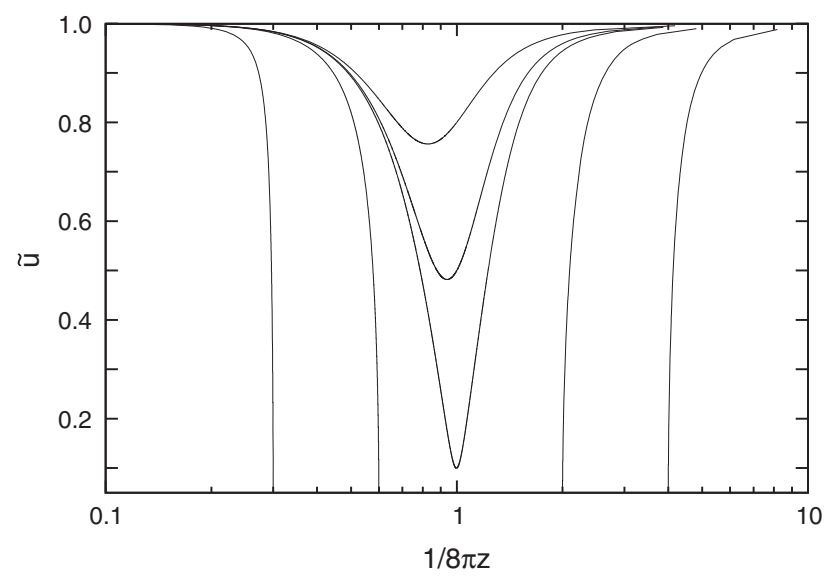

FIG. 1. The phase structure of the sine-Gordon model is presented. At $\tilde{u}=0$, we have a line of fixed points. At $\tilde{u}=1$ and $z \rightarrow 0 \quad(z \rightarrow \infty)$, we have an infrared (ultraviolet) nonGaussian fixed point, respectively. 
according to $\omega=\sqrt{1-\tilde{u}^{2}}, \chi=1 / z \omega$, and $\partial_{\tau}=\omega^{2} k \partial_{k}$ results in the evolution equations

$$
\begin{aligned}
& \partial_{\tau} \omega=2 \omega\left(1-\omega^{2}\right)-\frac{\omega^{2} \chi}{2 \pi}(1-\omega), \\
& \partial_{\tau} \chi=\chi^{2} \frac{1-\omega^{2}}{24 \pi}-2 \chi\left(1-\omega^{2}\right)+\frac{\omega \chi^{2}}{2 \pi}(1-\omega) .
\end{aligned}
$$

Here the fixed point $\chi^{*}=0$ and $\omega^{*}=0$ can be identified by the IR fixed point at $1 / z^{*}=0$ and $\tilde{u}^{*}=1$, which is IR attractive. Unfortunately, one cannot find this fixed point from the original evolution equations in Eq. (6) since it is situated at the singular point of the flow equation which makes the $\beta$ functions divergent. It might raise the question whether the singularity is a numerical artifact due to the truncations in the Fourier and in the gradient expansion or if it really exists. However, in $d=3$, we showed that the IR fixed point exists asymptotically if we do not use any truncation [31]. In the IR fixed point, the soft modes play a crucial role in the spontaneously broken phase and seem to bring the quantum system to a classical one. The huge amount of soft modes signals that the original degrees of freedom are not suitable to describe the low energy behavior of the SG model.

The problem of the irrelevant scaling in the symmetric phase can lead to divergences which can be avoided by finding a new nontrivial non-Gaussian fixed point in the UV region. This is the main idea of asymptotic safety. In this case, a residual interaction remains in the UV NGFP; therefore, it can be called an interacting fixed point. The concept of asymptotic safety can be considered as the generalization of the asymptotic freedom. The latter means that as we go to higher energies, the interaction becomes weaker and weaker. This property enables us to test the quantum chromodynamics with high energy collisions. However, there are models which have no UV attractive GFP. Then, either it might turn out that the model is only a part of a more fundamental theory, or the model has an UV NGFP; nevertheless, both cases can save the asymptotic behavior of the model. The former case can be exemplified by the four-dimensional (4D) $\phi^{4}$ theory, which, as part of the Standard Model (SM) describes the Higgs field, but its GFP is a saddle point; therefore, the model is perturbatively nonrenormalizable. However, according to the idea of the global renormalization group [12], the trajectory in the SM evolves among the fixed points of the model as the energy scale changes. As the flow approaches one fixed point, then the irrelevant coupling in its vicinity pushes away the trajectory towards another fixed point. This happens in the neighborhood of the GFP of the Higgs field. Presumably, the SM or its generalization contains high energy fixed points which save the model from divergences that appear seemingly in the pure $4 \mathrm{D} \phi^{4}$ theory.

The UV NGFP keeps the value of the couplings and, thus, the physical quantities finite. From the phase space in
Fig. 1, it is obvious that the fundamental mode $\tilde{u}$ does not diverge, but it tends to 1 as $z$ tends to 0 in the UV limit. This motivated the choice of the logarithmic scaling in $z$. Unfortunately, the limit $\tilde{u} \rightarrow 1$ makes the RG equations in Eq. (6) singular as in the IR limit. Thus, we should approach the UV region of the SG model in a similar manner, i.e., by a redefinition of the couplings. In the UV limit, the rescalings $\omega=\sqrt{1-\tilde{u}^{2}}, \zeta=z \omega$, and $\partial_{\tau}=$ $z \omega^{2} k \partial_{k}$ result in the evolution equations

$$
\begin{aligned}
& \partial_{\tau} \omega=2 \zeta \omega\left(1-\omega^{2}\right)-\frac{\omega^{2}}{2 \pi}(1-\omega), \\
& \partial_{\tau} \zeta=\left(2 \zeta^{2}-\frac{\zeta}{24 \pi}\right)\left(1-\omega^{2}\right)-\frac{\omega \zeta}{2 \pi}(1-\omega) .
\end{aligned}
$$

Although the UV NGFP cannot be seen directly analytically from Eq. (6), the transformed equations in Eq. (8) contain the corresponding fixed point at $\omega^{*}=0$ and $\zeta^{*}=0$. This result can be affirmed by the fact that the ratio $d u / d z$ calculated from the RG equations in Eq. (6) tends to zero if $z \rightarrow 0$ and $\tilde{u} \rightarrow 1$. In Fig. 2 , the evolution of the couplings $\tilde{u}$ and $z$ is shown. In the IR limit, both couplings diverge at a certain value of $k_{c}$, which defines the IR correlation length. In the UV limit, the coupling $\tilde{u}$ tends to 1, making the evolution equations in Eq. (6) singular. In Fig. 2, it is also shown that in the UV limit, the coupling $z$ tends to zero; therefore, the kinetic term in the action freezes out, and only the potential term remains. A similar situation appears in the confinement mechanism. Although the couplings change abruptly at $k_{c}$, the trajectories do not show a singular behavior, since the sudden increase of $\tilde{u}$ and the sudden decrease of $z$ compensate each other, giving regular flows. We obtained that in the vicinity of the UV NGFP, the numerically calculated scaling gives $z=(1-\tilde{u})^{3 / 2}$. The singular behavior in the UV limit defines a corresponding correlation length $\xi=1 / k_{c}$. The

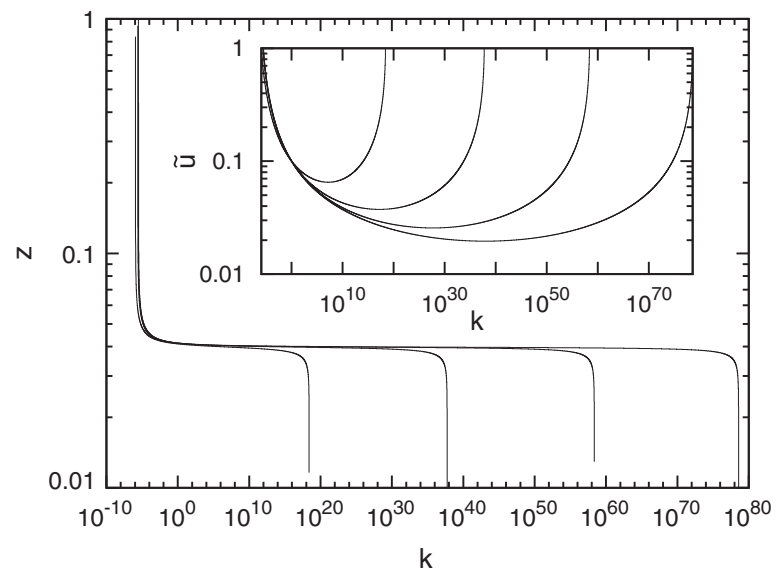

FIG. 2. The evolution of the couplings $\tilde{u}$ and $z$ is shown. In the IR and the UV limits, the flows abruptly grow up or fall down at the same scale $k$. 
reduced temperature can be identified as the deviation of the initial value of $z(\Lambda)$ to its critical value, i.e., $t \sim z(\Lambda)^{*}-z(\Lambda)$. For the KT-type phase transition, we have

$$
\log \xi \propto t^{-\nu}
$$

We determined the stopping scales $k_{c}$ for different initial couplings $z(\Lambda)$, and the numerical values gave $\nu \approx 0.51$ for the SG model. This implies that the essential scaling can be uncovered in the UV limit, too, although it originates from the slowing down of the flows around the KT point.

The point $z=0, \tilde{u}=1$ of the phase space corresponds to the UV NGFP of the 2D SG model, and it also makes the RG equations singular. The UV singularity seems to mark the upper limit of the applicability of the SG model. At higher energies, the model needs new elementary excitations. Thinking in the framework of the 2D XY model, the excitations are represented by vortices which are made up by concentric forms of spins [58]. The blocking towards the increasing value of the scale $k$ results in smaller and smaller vortices. At a certain scale $k_{c}$, the vortex reduces to a single spin. This scale can be identified by the scale in the UV limit where the $\beta$ functions become singular. At this scale, the new elementary excitations should be the single spins instead of the original vortices, implying that in the UV limit, the original degrees of freedom of charged vortices should be replaced by a neutral spin system.
The high energy behavior around the interacting fixed point can hardly be tested experimentally; nevertheless, there are attempts to support QEG with indirect or direct effects of gravity even at the LHC processes $[59,60]$. The situation is even worse if we consider the SG model, since it is not a fundamental theory. It is used mainly for treating condensed matter systems, where the energy scales are quite limited; therefore, the regions near the UV and IR fixed points cannot be reached by experimenters. This fact suggests that the asymptotically safe nature of the SG model cannot be experimentally tested.

However, the asymptotic safety has a theoretical importance, since it clarifies the UV scaling behavior of the model. Then, the functional RG method provides us both the high and low energy-scale limits of the applicability of the SG model. The low energy limit is usually indicated by the IR fixed point in the broken phase. There, the appearing condensate signals the necessity of the new degrees of freedom. Anyway, every model should also have an upper UV limit of its validity, since presumably, new degrees of freedom should appear at higher and higher energies.

\section{THE SINE-GORDON MODEL WITH AN IRRELEVANT COUPLING}

Let us include the running of the coupling $\tilde{z}_{1}$ in the SG model; we abbreviate it as the ZSG model. Then, the RG equations for the dimensionless couplings become

$$
\begin{aligned}
\dot{\tilde{u}}= & -2 \tilde{u}-\frac{1}{\tilde{u}} \int_{y}\left[1-\frac{\tilde{Z} y+1}{\left[(\tilde{Z} y+1)^{2}-\tilde{u}^{2}\right]^{1 / 2}}\right], \\
\dot{z}= & \frac{\tilde{u}^{2}}{4} \int_{y}\left[\frac{-\left(2 \partial_{y} \tilde{Z}+4 \tilde{z}_{1} y\right)(\tilde{Z} y+1)}{\left[(\tilde{Z} y+1)^{2}-\tilde{u}^{2}\right]^{5 / 2}}+\frac{y\left(\partial_{y} \tilde{Z}\right)^{2}\left(4(\tilde{Z} y+1)^{2}+\tilde{u}^{2}\right)}{\left[(\tilde{Z} y+1)^{2}-\tilde{u}^{2}\right]^{7 / 2}}\right], \\
\dot{\tilde{z}}_{1}= & 2 \tilde{z}_{1}+\frac{1}{48} \int_{y}\left[\frac{-24 \tilde{z}_{1}(\tilde{Z} y+1)}{\left[(\tilde{Z} y+1)^{2}-\tilde{u}^{2}\right]^{5 / 2}}+\frac{\left(72 \tilde{z}_{1}\left(\partial_{y} \tilde{Z}\right) y+6\left(\partial_{y} \tilde{Z}\right)^{2}+36 \tilde{z}_{1}^{2} y^{2}\right)\left(4\left(1+z y+\tilde{z}_{1} y^{2}\right)^{2}+\tilde{u}^{2}\right)}{\left[(\tilde{Z} y+1)^{2}-\tilde{u}^{2}\right]^{7 / 2}}\right. \\
& \left.+\frac{\left(-36\left(\partial_{y} \tilde{Z}\right)^{3} y-108 z_{1}\left(\partial_{y} \tilde{Z}\right)^{2} y^{2}\right)(\tilde{Z} y+1)\left(4(\tilde{Z} y+1)^{2}+3 \tilde{u}^{2}\right)}{\left[(\tilde{Z} y+1)^{2}-\tilde{u}^{2}\right]^{9 / 2}}\right], \\
& \left.+\frac{\left(18\left(\partial_{y} \tilde{Z}\right)^{4} y^{2}\right)\left(8(\tilde{Z} y+1)^{4}+12(\tilde{Z} y+1)^{2} \tilde{u}^{2}+\tilde{u}^{4}\right)}{\left[(\tilde{Z} y+1)^{2}-\tilde{u}^{2}\right]^{11 / 2}}\right]
\end{aligned}
$$

with $\tilde{Z}=z y+\tilde{z}_{1} y^{2}$. The momentum integral over $y=p^{2}$ cannot be performed analytically. Although the $\beta$ function for the coupling $\tilde{z}_{1}$ is quite involved, the numerical results show that the term coming from the inverse mass dimension of $\tilde{z}_{1}$ drives the RG flow according to

$$
\tilde{z}_{1} \sim k^{2}
$$

giving an irrelevant scaling in the IR limit. It implies that in the IR limit, the inclusion of $\tilde{z}_{1}$ does not affect the scaling of the SG model. There, the phase transition remains a KT-type transition, and we have the same phase structure as in Fig. 1. However, in the opposite scaling direction towards the UV limit, $\tilde{z}_{1}$ diverges, and it removes the UV NGFP. The phase space of the ZSG model is sketched in Fig. 3 for UV flows. In Eq. (10), the RG equations become singular if the denominator

$$
(\tilde{Z} y+1)^{2}-\tilde{u}^{2}=0
$$




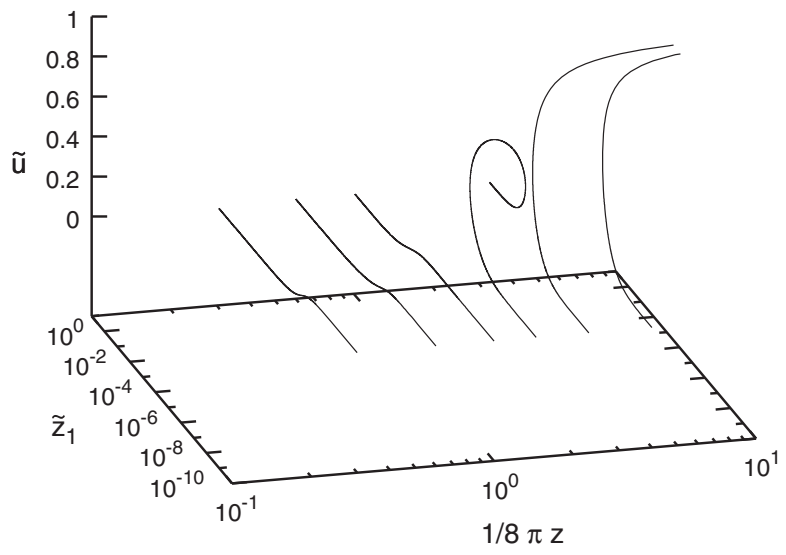

FIG. 3. The UV tendencies are plotted for the ZSG model with the choice $\tilde{z}_{1}(\Lambda)=0.0$. The coupling $\tilde{z}_{1}$ tends to infinity except when the singularity prevents it.

for any momentum. It can be zero if $\tilde{u} \rightarrow 1$. However, the coupling $\tilde{z}_{1}$ grows up fast, which can prevent the singularity. The competition of these two scalings creates such trajectories which constitute the two phases of the model. In Fig. 3, the UV symmetric phase contains such trajectories where $\tilde{z}_{1} \rightarrow \infty$. The others will stop at finite $k$ and belong to the broken phase. The UV correlation length can be defined similarly to the IR one, i.e., via the stopping scale of $k_{c}$ where the UV evolution stops. Its scaling shows that instead of the essential scaling, there occurs a second order Ising-type phase transition according to

$$
\xi \sim t^{-\nu}
$$

with the exponent $\nu=1 / 4$. If $\tilde{z}_{1}(\Lambda)$ is increased, then the broken phase starts to shrink. The large initial value of $\tilde{z}_{1}$ can even overwrite the scaling behavior of $\tilde{u}$ in the way that the original starting relevant scaling for $1 / z>8 \pi$ will be irrelevant and keeps the trajectories in the UV symmetric phase. It is worth noting that a starting UV relevant scaling of $\tilde{u}$ can turn to irrelevant (the middling trajectory in Fig. 3). Such a flow did not appear in the original SG model.

The inclusion of the new coupling $\tilde{z}_{1}$ does not give a new phase. One can check it with the sensitivity matrix $[12,55]$, which characterizes the deviation of the running coupling with respect to the bare ones. According to Fig. 3, certain trajectories go to infinity in the UV, and there are ones which stop at a finite scale $k$. They can be neighboring trajectories which are adjacent in the IR limit and then build up infinitely large distances from each other in the UV limit, signaling distinct phases according to the sensitivity matrix. There are no further trajectories which can develop further singularities, implying that we have no additional phases in the ZSG model.

\section{THE SINE-GORDON MODEL WITH A RELEVANT COUPLING}

If we include a mass term in the potential

$$
V_{k}(\phi)=\frac{1}{2} \tilde{m}^{2} \phi^{2}+\tilde{u} \cos (\phi),
$$

then we have modified the SG model which loses its periodic symmetry. We call it the MSG model. Neglecting the evolution of $\tilde{z}_{1}$, the RG equations are $[55,56]$

$$
\begin{aligned}
\dot{\tilde{u}} & =-2 \tilde{u}+\frac{1}{2 \pi \tilde{u} z}\left[1+\tilde{m}^{2}-\sqrt{\left(1+\tilde{m}^{2}\right)^{2}-\tilde{u}^{2}}\right], \\
\dot{z} & =-\frac{1}{24 \pi} \frac{\tilde{u}^{2}}{\left(\left(1+\tilde{m}^{2}\right)^{2}-\tilde{u}^{2}\right)^{3 / 2}}, \\
\dot{\tilde{m}}^{2} & =-2 \tilde{m}^{2} .
\end{aligned}
$$

The mass $\tilde{m}^{2}$ decouples from the other couplings, and it scales according to the mass dimension of $\mathrm{m}^{2}$,

$$
\tilde{m}^{2} \sim k^{-2},
$$

so it is a relevant coupling as opposed to the previously introduced coupling $\tilde{z}_{1}$. In the IR limit, the MSG model shows a second order phase transition. There, we got $\nu=$ $1 / 2$ [30] for the exponent of the correlation length in Eq. (13). In the IR limit, the IR fixed point vanishes, since the mass scales in a relevant manner there. In the UV limit, the mass dies out due to its relevant scaling; therefore, the MSG and the SG models coincide, and, consequently, it shows a KT-type infinite order phase transition.

\section{DUALITY}

If we consider the phase diagram of the SG model with the couplings $\tilde{u}$ and $z$ in Fig. 1, then we can realize that the UV and the IR limits take place in a quite symmetric manner there. If we make the following changes

$$
\begin{aligned}
k & \rightarrow \frac{1}{k}, \\
z & \rightarrow \frac{1}{z}
\end{aligned}
$$

in the flow direction of the evolution (this is how the UV limits are usually investigated) and the coupling, then we obtain a qualitatively similar phase structure. The first relation in Eq. (17) can be interpreted as a RG time reversal. Similar relations were treated in [58]. In this sense, the SG model shows a self-duality. There are trajectories that belong to the broken phase and start from the vicinity of the UV NGFP and then they flow into the IR fixed point. According to the self-duality, these types of trajectories flow from the IR fixed point to the UV one if the RG time is reversed. 
TABLE I. Summary of the SG-type models and their fixed points.

\begin{tabular}{lcc}
\hline \hline Model & UV & IR \\
\hline SG & KT type, $\nu=1 / 2$ & KT type, $\nu=1 / 2$ \\
MSG & KT type, $\nu=1 / 2$ & Ising type, $\nu=1 / 2$ \\
ZSG & Ising type, $\nu=1 / 4$ & KT type, $\nu=1 / 2$ \\
\hline \hline
\end{tabular}

In the UV region, the coupling $\tilde{u}$ is irrelevant, and it turns to relevant in the IR limit. Its large value in the UV rapidly decreases around the KT point. Going along the evolution towards the IR regime, the tendency of the flow changes, and the value of the coupling starts to grow up. The fast increase implies that the flow in the IR limit may enlarge very small effects which are picked up during the evolution even in the vicinity of the UV NGFP. This is the idea of the RG microscope. It implies that the UV and the IR fixed points interact.

The coupling $\tilde{u}$ of the SG model scales in an irrelevant manner around the KT point, but it cannot be canceled out since it is the only crucial parameter in the theory. The UV NGFP saves the SG model and makes it meaningful in the $\mathrm{UV}$ region. The same mechanism takes place in QEG where the Newton coupling is irrelevant around the GFP but scales in a relevant manner around the UV NGFP.

Physically, the broken phase of the SG model can be considered as the phase of bound vortex pairs in the equivalent 2D XY model. The small (large) value of the coupling $\tilde{u}$ corresponds to strong (weak) interaction between the vortex-antivortex pairs, respectively. Thus, the irrelevant scaling in the UV implies growing vortex interactions, and after the turn of the flow in the vicinity of the KT point, the irrelevant scaling signals the weakening of the vortex-antivortex binding. The maximal value of $\tilde{u}$ in the UV and IR limits means that there is a lower bound for the strength of the vortex interactions. In the IR fixed point, the whole system contains only one gigantic vortex pair, while in the UV limit, the system disintegrates into single spins. According to the self-duality, these limiting situations constitute a dual pair. It clearly shows that these limitations of the XY model require the necessity of introducing new degrees of freedom, as in the case of the equivalent $\mathrm{SG}$ model.

The advantage of the duality connections is that it is enough to investigate a certain limitation of the model, and we can conclude the properties of the other limit from the former results. The UV and the IR fixed points create a dual pair, and the lines of fixed points separated by the KT point turn from one to the other under the dual transformation in Eq. (17). The KT fixed point remains unchanged.

The duality can be extended to the ZSG and to the MSG models, too. In the former model, the original SG model contains a further irrelevant coupling, while the MSG model has an additional relevant one. We collected the appearing types of phase transitions in these models in Table I. In the ZSG model, the IR scalings are not affected by irrelevant couplings. The same is true with opposite flow directions for the MSG model; there, the UV scaling remains unchanged due to the new relevant coupling. It implies that the IR ZSG and the UV MSG models behave as the original SG model; they show an essential scaling. However, in the other directions, we have significant changes. In the UV limit of the ZSG model and in the IR limit of the MSG model, we can identify second order phase transitions. It means that by completing the dual transformations in Eq. (17) with

$$
\tilde{z}_{1} \rightarrow \tilde{m}^{2}
$$

then the ZSG and the MSG models become a dual pair. It is not obvious that there exist any relations between these models. The MSG model is the bosonized version of the 2D quantum electrodynamics (or Schwinger model) [54-56,61] and has no direct relation to the original SG model since it is well known that after the bosonization it becomes the massive Thirring model [1]. The ZSG model can be related to the latter one, since there the wave-function renormalization is treated in a more precise way.

\section{SUMMARY}

The 2D SG model and its extensions have been investigated by the functional renormalization group method taking care of the deep IR and the far UV limits. We found that the SG model is asymptotically safe; i.e., it possesses a NGFP in the UV limit. The UV NGFP is hidden by the singularity which nicely traces out the upper limitations of the model. The singularity has been identified in the IR limit, too. The similar behavior raises the question of the self-duality of the SG model for the UV and the IR limits.

We extended the SG model by taking into account the evolution of a further coupling in the momentum-dependent wave-function renormalization. We showed that the obtained ZSG model has no UV NGFP, and the UV critical behavior is an Ising-type phase transition. It reminds us of the MSG model, where the IR limits showed a second order phase transition. This fact uncovered the dual relation between the ZSG and the MSG models.

\section{ACKNOWLEDGMENTS}

This research was supported by the European Union and the State of Hungary, cofinanced by the European Social Fund in the framework of the TÁMOP 4.2.4.A/2-11-12012-0001 National Excellence Program. S. N. acknowledges financial support from a János Bolyai Grant of the Hungarian Academy of Sciences and the Hungarian National Research Fund OTKA (K112233). 
[1] S. R. Coleman, Phys. Rev. D 11, 2088 (1975).

[2] L. Benfatto, C. Castellani, and T. Giamarchi, Phys. Rev. Lett. 99, 207002 (2007).

[3] Z. Bajnok, L. Palla, and G. Takacs, Nucl. Phys. B644, 509 (2002).

[4] Z. Bajnok, C. Dunning, L. Palla, G. Takacs, and F. Wagner, Nucl. Phys. B679, 521 (2004).

[5] D. M. Hofman and J. M. Maldacena, J. Phys. A 39, 13095 (2006).

[6] A. B. Zamolodchikov, Int. J. Mod. Phys. A 10, 1125 (1995).

[7] G. Delfino and G. Mussardo, Nucl. Phys. B516, 675 (1998).

[8] G. Mussardo, V. Riva, and G. Sotkov, Nucl. Phys. B687, 189 (2004).

[9] A. Codello, G. D’Odorico, and C. Pagani, J. High Energy Phys. 07 (2014) 040.

[10] C. Wetterich, Phys. Lett. B 301, 90 (1993).

[11] J. Berges, N. Tetradis, and C. Wetterich, Phys. Rep. 363, 223 (2002).

[12] J. Polonyi, Central Eur. J. Phys. 1, 1 (2003).

[13] J. M. Pawlowski, Ann. Phys. (Amsterdam) 322, 2831 (2007).

[14] H. Gies, Lect. Notes Phys. 852, 287 (2012).

[15] V. L. Berezinskii, Sov. Phys. JETP 34, 610 (1972).

[16] J. Kosterlitz and D. Thouless, J. Phys. C 6, 1181 (1973).

[17] I. Nandori, J. Polonyi, and K. Sailer, Phys. Rev. D 63, 045022 (2001)

[18] S. Nagy, I. Nandori, J. Polonyi, and K. Sailer, Phys. Lett. B 647, 152 (2007).

[19] V. Pangon, S. Nagy, J. Polonyi, and K. Sailer, Phys. Lett. B 694, 89 (2010).

[20] D. J. Amit, Y. Y. Goldschmidt, and G. Grinstein, J. Phys. A 13, 585 (1980).

[21] M. Grater and C. Wetterich, Phys. Rev. Lett. 75, 378 (1995).

[22] G. Von Gersdorff and C. Wetterich, Phys. Rev. B 64, 054513 (2001).

[23] S. Nagy, I. Nandori, J. Polonyi, and K. Sailer, Phys. Rev. Lett. 102, 241603 (2009).

[24] S. Nagy and K. Sailer, Int. J. Mod. Phys. A 28, 1350130 (2013).

[25] I. Nandori, arXiv:1108.4643.

[26] N. Tetradis and C. Wetterich, Nucl. Phys. B383, 197 (1992).

[27] D. B. Kaplan, J.-W. Lee, D. T. Son, and M. A. Stephanov, Phys. Rev. D 80, 125005 (2009).

[28] J. Braun, H. Gies, and D. D. Scherer, Phys. Rev. D 83, 085012 (2011).

[29] S. Nagy, Phys. Rev. D 86, 085020 (2012).
[30] S. Nagy, Nucl. Phys. B864, 226 (2012).

[31] S. Nagy, Ann. Phys. (Amsterdam) 350, 310 (2014).

[32] I. Ya. Arefeva and V. E. Korepin, JETP Lett. 20, 312 (1974).

[33] A. B. Zamolodchikov and A. B. Zamolodchikov, Ann. Phys. (N.Y.) 120, 253 (1979).

[34] E. Sklyanin, J. Sov. Math. 47, 2473 (1989).

[35] E. Sklyanin, J. Phys. A 21, 2375 (1988).

[36] S. Ghoshal and A. B. Zamolodchikov, Int. J. Mod. Phys. A 09, 3841 (1994).

[37] Z. Bajnok, L. Palla, G. Takacs, and G. Toth, Nucl. Phys. B622, 548 (2002).

[38] Z. Bajnok, L. Palla, and G. Takacs, Proc. Sci., unesp2002 (2002) 004 [arXiv:hep-th/0211132].

[39] V. Pangon, arXiv:1111.6425.

[40] R. Percacci, Proc. Sci., CLAQG08 (2011) 002 [arXiv: 0910.4951].

[41] A. Zamolodchikov, JETP Lett. 43, 730 (1986).

[42] C. Rim, arXiv:hep-th/0405162.

[43] R. Percacci, arXiv:0709.3851.

[44] M. Reuter and F. Saueressig, arXiv:0708.1317.

[45] D. F. Litim and F. Sannino, J. High Energy Phys. 12 (2014) 178.

[46] M. Reuter, Phys. Rev. D 57, 971 (1998).

[47] M. Reuter and F. Saueressig, Phys. Rev. D 65, 065016 (2002).

[48] M. Reuter and F. Saueressig, New J. Phys. 14, 055022 (2012).

[49] A. Eichhorn and H. Gies, New J. Phys. 13, 125012 (2011).

[50] S. Rechenberger and F. Saueressig, Phys. Rev. D 86, 024018 (2012).

[51] A. Eichhorn, Phys. Rev. D 86, 015021 (2012).

[52] P. Don, A. Eichhorn, and R. Percacci, Phys. Rev. D 89, 084035 (2014).

[53] A. Eichhorn, Phys. Rev. D 87, 124016 (2013).

[54] S. Nagy, J. Polonyi, and K. Sailer, Phys. Rev. D 70, 105023 (2004).

[55] S. Nagy, I. Nandori, J. Polonyi, and K. Sailer, Phys. Rev. D 77, 025026 (2008).

[56] I. Nandori, Phys. Rev. D 84, 065024 (2011).

[57] I. Nandori, J. High Energy Phys. 04 (2013) 150.

[58] K. Huang and J. Polonyi, Int. J. Mod. Phys. A 06, 409 (1991).

[59] B. Dobrich and A. Eichhorn, J. High Energy Phys. 06 (2012) 156.

[60] A. Eichhorn, arXiv:1210.1528.

[61] I. Marian, U. Jentschura, and I. Nandori, J. Phys. G 41, 055001 (2014). 